\title{
Avaliação dos Esquemas Iniciais Desadaptativos: estudo psicométrico em alcoolistas
}

\author{
Evaluation of Early Maladaptive Schemas: psychometric study in alcoholics \\ Jaqueline Garcia da Silva', Milton José Cazassa², Margareth da Silva Oliveiraª, Gabriel Chittó Gauer ${ }^{4}$
}

\author{
Palavras-chave \\ Alcoolismo, Esquemas \\ Iniciais Desadaptativos, \\ Young Schema \\ Questionnaire, \\ personalidade.
}

\begin{abstract}
RESUMO
Objetivo: Este estudo objetiva apresentar as propriedades psicométricas do Questionário de Esquemas de Young - forma reduzida (YSQ-S2). Método: Foi realizado um estudo quantitativo, transversal, comparando um grupo clínico de alcoolistas com um grupo não clínico. Os resultados foram analisados com o alfa de Cronbach, o teste t de Student e a Análise Discriminante. A amostra da pesquisa foi constituída por 107 sujeitos, sendo 51 alcoolistas e 56 de uma amostra populacional do grupo de não alcoolistas. Resultados: Foram encontradas diferenças estatisticamente significativas nas cinco áreas e nos 15 esquemas avaliados pelo questionário YSQ-S2 quando comparados os grupos clínico e não clínico. Conclusão: Foi observado que o YSQ-S2 é um instrumento capaz de detectar diferenças entre populações clínicas e não clínicas, sendo um importante recurso para uma avaliação mais profunda do funcionamento da personalidade do paciente.
\end{abstract}

\begin{abstract}
Objective: This study aims at presenting the psychometric properties of the Young Schema Questionnaire - short form (YSQ-S2). Method: We conducted a quantitative study, cross-sectional comparing a clinical with a non-clinical group and Cronbach's alpha, $\mathrm{t}$ test Student and Discriminant Analysis were used to analyze the results. The sample consisted of 107 subjects, 51 alcoholics and 56 non-alcoholics. Results: Statistically significant differences were found in the five areas and the 15 schemes assessed by the YSQ-S2 questionnaire when comparing the clinical and non-clinical groups. Conclusion: YSQ-S2 was found to be an instrument that can detect differences between clinical and non-clinical populations, its use being essential for a deeper assessment of the patient's personality.
\end{abstract}

\section{Keywords}

Alcoholism, Early

Maladaptive Schemas,

Young Schema

Questionnaire, personality. profundo da cognição originalmente chamado de Early Maladaptive Schemas (EMS) e traduzido como Esquemas Iniciais Desadaptativos (EID). Young ${ }^{1,2}$ ampliou o modelo original da terapia cognitiva para a identificação dos EID, os quais propiciam que o indivíduo crie padrões de compreensão distorcidos em relação ao mundo, a ele próprio e aos outros.
A Terapia Focada em Esquemas tem como precursores Young, Klosko e Weishaar e representa uma evolução do modelo cognitivo de Aaron Beck para o tratamento de transtornos de personalidade, com a ênfase em um nível mais
1 Universidade Católica do Rio Grande do Sul (PUCRS); Universidade de Granada, Espanha. 2 PUCRS.

Recebido em 20/6/2012 Aprovado em $8 / 10 / 2012$ 4 PUCRS, Grupo de Pesquisa, Avaliação e Intervenção em Saúde Mental.
3 Universidade Federal de São Paulo (Unifesp); PUCRS, Grupo de Pesquisa, Avaliação e Atendimento em Psicoterapia Cognitiva.

Endereço para correspondência: Margareth da Silva Oliveira Pontifícia Universidade Católica do Rio Grande do Sul Av. Ipiranga, 6681, prédio 11, sala 927, Partenon - 90619-900 - Porto Alegre, RS, Brasil E-mail: marga@pucrs.br 
Os EID são estruturas estáveis e duradouras que se desenvolvem e se cristalizam na personalidade ao longo da vida, configurando-se como padrões amplos, formados por memórias, emoções e sensações corporais, relacionadas à pessoa ou à maneira como se relaciona com os outros ${ }^{3}$. Apesar de nem todos os esquemas possuírem traumas em sua origem, esses padrões de funcionamento são destrutivos e causadores de sofrimento 4,5 .

Para Soygüt et al. ${ }^{6}$, os esquemas, geralmente, se desenvolvem a partir das primeiras experiências com as figuras parentais e, na maioria das vezes, são causados pela vivência de experiências traumáticas que se repetem com alguma regularidade no decorrer da vida e que impossibilitam a satisfação de necessidades emocionais essenciais do ser humano, entre elas o vínculo seguro com outras pessoas, incluindo proteção, estabilidade e segurança; autonomia, competência e senso de identidade; liberdade para expressar necessidades e emoções; espontaneidade e diversão; limites precisos e autocontrole. Dessa forma, os esquemas encontram-se vinculados a diversos transtornos mentais.

Com o objetivo de identificar os EID, Young et al. ${ }^{3}$ construíram o Young Schema Questionnaire, o qual já possui, além da versão original (205 itens), a versão reduzida (75 itens). Neste estudo foi utilizada a versão reduzida por ela apresentar excelente grau de confiabilidade na validação brasileira para população geral (alfa 0,95$)^{78}$. Não foram encontrados mais artigos com estudos clínicos, assim sendo este estudo é o primeiro no Brasil realizando comparações entre amostras clínicas e não clínicas.

O questionário está estruturado em cinco domínios, nos quais os esquemas foram agrupados a partir da experiência clínica do autor com pacientes crônicos que não evoluíam na psicoterapia. Esses domínios originais foram refinados em um estudo empírico realizado por Schmidt et al. ${ }^{5}$, e outros estudos foram conduzidos em diversos países ${ }^{9-13}$. O instrumento contempla a possibilidade de verificação de processos de reforçamento dos esquemas, tais como distorções cognitivas e padrões de comportamentos autoderrotistas 5 .

Uma vez identificados, os EID podem ser objetivamente trabalhados com intervenções específicas desenvolvidas para as distorções cognitivas e redução dos sintomas relatados $^{14-16}$. Em diferentes países e amostras, O YSQ-S2 tem sido administrado, como no estudo realizado com dependentes de álcool, no qual os resultados indicam que o número de internações foi negativamente correlacionado com o padrão de privação emocional e positivamente correlacionado com o padrão de isolamento social ${ }^{17}$. Os autores entenderam a primeira correlação pelo fato de que no hospital os pacientes podem romper o isolamento, pois se encontram inseridos em um grupo (e afirmam sentir-se bem), ou seja, estão com pessoas que têm "o mesmo problema". Por outro lado, estar hospitalizado poderia ser uma oportunidade para a reativação do esquema de isolamento, já que o padrão de privação emocional é esperado na internação, na medida em que o sujeito pode pensar que os outros pacientes não podem ajudá-lo e, dessa forma, poderá não receber o apoio de que necessita e até pensar que estar hospitalizado não vai Ihe ajudar ${ }^{17}$. Os esquemas pontuados significativamente foram abandono ( $p=0,001)$, autossacrifício $(p=0,001)$ e vulnerabilidade $(p=0,001)$. Correlações positivas foram obtidas entre a estratégia de prevenção e a pontuação total de padrões de ativação $(r=0,48)$ com dois esquemas: abandono $(r$ $=0,60)$ e vulnerabilidade a dores e doenças $(r=0,60)$. Correlações negativas foram obtidas entre a busca de apoio social e inibição emocional $(r=-0,72)$ e merecimento $(r=-0,50)^{17}$.

Brotchie et al. ${ }^{18}$ compararam uma amostra clínica de usuários ( $n=97$ ) de álcool, de opiáceos e de álcool e opiáceos combinados com uma amostra não clínica $(n=87)$. Todos os três grupos clínicos tinham esquemas disfuncionais na comparação com o grupo não clínico, mas o tema dessas cognições diferiu entre os grupos clínicos. O grupo abusador de álcool foi caracterizado por um maior nível de distorções cognitivas. O grupo de somente usuários de álcool ou combinado com opiáceos foi associado mais fortemente do que o grupo de abuso de opiáceos com três crenças negativas (vulnerabilidade ao dano, subjugação e inibição emocional). Esses dados refletem uma tentativa de lidar com um padrão de falhas pessoais percebidas, que são maiores nos abusadores de álcool do que nos sujeitos abusadores de opiáceos apenas. Para obter os resultados, foram realizadas análises post hoc com o valor de $\mathrm{p}<0,01$ para todas as diferenças indicadas. As conclusões apontaram para o fato de o álcool ser utilizado para reduzir a experiência do afeto negativo, uma vez que esta foi ativada. Os autores sugerem a continuidade de pesquisas com populações clínicas e não clínicas.

Neste estudo, além de serem apresentados os dados referentes aos EID nas populações clínica e não clínica, também se tem como objetivo apresentar dados referentes à validação discriminante do YSQ-S2.

\section{MÉTODO}

Trata-se de uma pesquisa transversal com metodologia quantitativa para avaliação da validade discriminante do instrumento. A amostra foi constituída por 107 participantes, sendo 51 alcoolistas, provenientes de clínicas e hospitais especializados para o tratamento do alcoolismo. Os 56 sujeitos da população não clínica foram recrutados em escolas e empresas, considerando-se que na entrevista eles negavam fazer uso de substâncias psicoativas, exceto nicotina.

Os critérios de inclusão na amostra foram vinculados à escolaridade e idade dos participantes. Foram incluídos no estudo participantes de 18 a 60 anos, com escolarização mínima de cinco anos de estudo, e foram excluídos os que apresentaram diagnóstico de transtornos mentais graves, a partir de dados do prontuário do paciente. Na amostra do grupo não 
clínico, foi considerado critério de exclusão o uso de substâncias psicoativas, como álcool, cocaína e anfetaminas, exceto o uso tabaco, que não foi considerado fator de exclusão.

\section{Instrumentos}

Os instrumentos utilizados foram os seguintes:

Questionário de Dados Sociodemográficos - Utilizado para mapear características sociodemográficas, tais como idade, sexo, escolaridade, estado civil, consumo de álcool, entre outras.

YSQ-S2 - Trata-se do Young Schema Questionnaire Short Form 2, traduzido como Questionário de Esquemas de Young - forma reduzida. O instrumento objetiva avaliar 15 esquemas considerados como centrais na cognição humana e, para tanto, é formado por 75 afirmativas, as quais são pontuadas de 1 a 6 de acordo com a percepção do examinando. Os 15 esquemas encontram-se inseridos em cinco grandes grupos, a saber:

1) Desconexão e rejeição: Diz respeito ao sentimento de frustração vivenciado pela pessoa com relação às expectativas de segurança, estabilidade, carinho, empatia, compartilhamento de sentimentos, aceitação e consideração. O questionário de Young avalia cinco esquemas que estão vinculados a esse grupo - privação emocional, abandono, desconfiança/ abuso, isolamento social e defectividade/vergonha.

2) Autonomia e desempenho prejudicados: Esquema Inicial Desadaptativo (EID), que configura sentimentos de incapacidade experimentados pelo indivíduo no que tange à possibilidade de se separar dos demais, conquistando a autonomia necessária para sobreviver de forma independente e com bom desempenho (os esquemas são fracasso, dependência/incompetência, vulnerabilidade a dores e doenças, emaranhamento).

3) Limites prejudicados: EID possível de ser identificado pela deficiência nos limites internos, pela ausência de responsabilidade com os demais e/ou pela dificuldade de orientação para a concretização de objetivos distantes. Caracteriza prejuízos com relação a respeitar os direitos dos outros, a cooperar e a se comprometer com metas ou desafios. Os esquemas associados a esse domínio são os de merecimento e autocontrole/autodisciplina insuficientes.

4) Orientação para o outro: Trata-se de um esquema que, quando presente, ocasiona um foco excessivo para os desejos e sentimentos dos outros por causa da constante busca de obtenção de amor. Muitas vezes, a pessoa suplanta suas próprias necessidades com o intuito de obter aprovação, podendo suprimir sua consciência, sentimentos e inclinações naturais. Os esquemas de subjugação e autossacrifício compõem esse grupo.

5) Supervigilância e inibição: Refere-se ao bloqueio da felicidade, autoexpressão, relaxamento, relacionamentos íntimos e ao comprometimento da própria saúde devido à ênfase excessiva na supressão dos sentimentos, dos impulsos e das escolhas pessoais espontâneas. Regras e expectativas rígidas internalizadas sobre desempenho e comportamento ético geralmente integram esse EID ${ }^{4}$. Inibição emocional e padrões inflexíveis são os dois esquemas que integram este domínio.

A coleta dos dados da população não clínica ocorreu em pessoas recrutadas em escolas e universidades. Na população clínica, a aplicação ocorreu em clínicas especializadas para tratamento do alcoolismo. A administração dos instrumentos foi individual.

O projeto foi submetido e aprovado pela Comissão Científica da Faculdade de Psicologia e Comitê de Ética em Pesquisa da PUCRS (CEP 07/03471) e os sujeitos participaram da pesquisa mediante o preenchimento do Termo de Consentimento Livre e Esclarecido. Os dados foram processados no programa estatístico SPSS 17.0 (Statistical Package for the Social Sciences). Foram realizadas análises descritivas, de distribuição de frequências para demonstração do perfil dos participantes da pesquisa, e a consistência interna foi avaliada pelo alfa de Cronbach, o teste t de Student e a Análise Discriminante para comparação entre os grupos.

\section{RESULTADOS}

A amostra foi constituída por 107 participantes do sexo masculino, sendo 51 alcoolistas e 56 sujeitos da população não clínica. A idade média foi de 42,73 $\pm 9,20$ anos, para a população não clínica, e de 45,07 \pm 9,62 anos, para os alcoolistas.

Em relação ao consumo de álcool, a população geral foi discriminada pela entrevista estruturada de dados sociodemográficos, que continha questões acerca do uso de álcool, frequência e tipo de bebida consumida e outras drogas (inclusive tabaco). Com relação ao consumo de drogas lícitas e ilícitas, na população não clínica, 15,7\% $(n=8)$ da amostra afirmaram fazer uso do cigarro, enquanto $84,3 \%(n=43)$ não usam a substância. No grupo de alcoolistas, $53,6 \%(n=30)$ fumavam e $46,4(n=26)$ referiram não fumar, sendo significativa essa diferença entre os grupos $(p=0,000)$. Quanto às drogas ilícitas, na população não clínica todos os participantes registraram não serem usuários de drogas. No grupo de alcoolistas, 23,2\% ( $n=$ 13) relataram haver usado cocaína alguma vez na vida, mas a internação se deu por causa da dependência de álcool.

No que se refere à realização de tratamento psicológico, o percentual apontou para o fato de que 92,0\% $(n=46)$ da população não clínica da pesquisa não realizavam tratamento psicológico, enquanto, no grupo de alcoolistas, 66,1\% (n = 37) estavam em processo psicoterapêutico no período do preenchimento do questionário de dados sociodemográfi$\cos (p=0,000)$. Além disso, 39,6\% ( $n=19)$ na população não clínica relataram usar medicação e no grupo de alcoolistas o percentual foi de $83,9 \%(n=47)(p=0,000)$.

A partir dos dados sociodemográicos na tabela 1, podese observar que ambos os grupos têm entre 9 e 12 anos ou 
mais de estudo (sendo essa uma variável que teve por objetivo parear para que não houvesse diferença, assim como a classificação socioeconômica, avaliada de acordo com o Critério de Classificação Econômica Brasil). Destacam-se os dados referentes à alta prevalência do uso de tabaco na população clínica, sendo também importante considerar outras características da amostra como não estar trabalhando no momento atual e um índice maior para solteiros ou separados, o que pode indicar de certa forma prejuízos sociais e ocupacionais decorrentes do uso do álcool.

\section{Consistência interna do YSQ-S2}

O coeficiente alfa de Cronbach foi utilizado para verificação da consistência interna do Questionário de Esquemas de Young - forma reduzida (YSQ-S2), considerando-se os 15 esquemas e a escala global. A seguir seguem os resultados gerais e para as populações clínica e não clínica, respectivamente. Para os 75 itens, o alfa foi de 0,96, 0,93 e 0,96; para o domínio 1, foi de 0,91, 0,85 e 0,91; para o domínio 2, de 0,89, 0,79 e 0,92; para o domínio 3, de 0,87, 0,84 e 0,84; para o domínio 4, de 0,81, 0,74 e 0,83; e para o domínio 5, de 0,84, 0,71 e 0,90, o que sugere excelente grau de consistência interna. De acordo com Bisquerra et al. ${ }^{19}$, coeficientes superiores a 0,75 são considerados altos.

Tabela 1. Dados sociodemográficos

\begin{tabular}{|c|c|c|c|c|}
\hline \multirow{2}{*}{ Dados sociodemográficos } & \multicolumn{2}{|c|}{ Grupo clínico } & \multicolumn{2}{|c|}{ Grupo não clínico } \\
\hline & $\mathrm{n}$ & $\%$ & $\mathrm{n}$ & $\%$ \\
\hline \multicolumn{5}{|l|}{ Anos de estudo } \\
\hline 5 a 8 anos de estudo & 11 & 19,7 & 9 & 17,6 \\
\hline 9 a 11 anos de estudo & 26 & 46,4 & 20 & 33,3 \\
\hline Acima de 12 anos de estudo & 20 & 35,7 & 15 & 43,2 \\
\hline \multicolumn{5}{|l|}{ Estado civil } \\
\hline Solteiro & 17 & 30,4 & 9 & 17,6 \\
\hline Casado ou com companheiro & 24 & 42,9 & 35 & 68,6 \\
\hline Viúvo & 2 & 3,6 & \multicolumn{2}{|c|}{ - } \\
\hline Separado ou divorciado & 13 & 23,2 & 7 & 13,7 \\
\hline \multicolumn{5}{|l|}{ Ocupação } \\
\hline Trabalha & 38 & 67,9 & 49 & 96,1 \\
\hline Não trabalha & 18 & 32,1 & 2 & 3,9 \\
\hline \multicolumn{5}{|l|}{ Hábito de fumar } \\
\hline Fuma & 30 & 53,6 & 8 & 15,7 \\
\hline Não fuma & 26 & 46,4 & 43 & 84,3 \\
\hline \multicolumn{5}{|l|}{ Renda mensal } \\
\hline Menor que $\mathrm{R} \$ 1.313,69$ & 11 & 19,6 & 21 & 41,2 \\
\hline De $R \$ 1.313,69$ a $R \$ 2.625,12$ & 27 & 48,2 & 13 & 25,5 \\
\hline Acima de $R \$ 2.625,12$ & 18 & 32,1 & 16 & 31,4 \\
\hline \multicolumn{5}{|l|}{ Classificação econômica } \\
\hline A & 4 & 7,2 & 1 & 2,0 \\
\hline B & 25 & 44,7 & 6 & 11,8 \\
\hline C & 18 & 32,1 & 22 & 43,1 \\
\hline$D / E$ & 9 & 16,1 & 22 & 42,2 \\
\hline
\end{tabular}

\section{Resultados do YSQ (S2)}

Os dados obtidos a partir da versão brasileira do Questionário de Esquemas de Young, forma reduzida (YSQ-S2), na amostra estudada, comparando grupo de alcoolistas e grupo não clínico pelo teste t de Student, permitiram encontrar diferenças estatisticamente significativas nos cinco domínios avaliados pelo YSQ e nos 15 esquemas que compõem o questionário. Os resultados são apresentados na tabela 2.

Tabela 2. Diferenças na comparação entre os grupos clínico e não clínico

\begin{tabular}{|c|c|c|c|}
\hline Esquemas/Domínios & Grupo & Média \pm DP & $t$ \\
\hline Abandono & $\begin{array}{l}\text { Não clínico } \\
\text { Clínico }\end{array}$ & $\begin{array}{l}1,89 \pm 0,95 \\
3,38 \pm 1,50\end{array}$ & 6,041 \\
\hline Desconfiança/Abuso & $\begin{array}{l}\text { Não clínico } \\
\text { Clínico }\end{array}$ & $\begin{array}{l}1,77 \pm 0,82 \\
2,92 \pm 1,16\end{array}$ & 5,839 \\
\hline Isolamento social & $\begin{array}{l}\text { Não clínico } \\
\text { Clínico }\end{array}$ & $\begin{array}{l}1,57 \pm 0,88 \\
2,33 \pm 1,02\end{array}$ & 4,070 \\
\hline Privação emocional & $\begin{array}{l}\text { Não clínico } \\
\text { Clínico }\end{array}$ & $\begin{array}{l}1,70 \pm 1,00 \\
2,56 \pm 1,26\end{array}$ & 3,879 \\
\hline Defectividade/Vergonha & $\begin{array}{l}\text { Não clínico } \\
\text { Clínico }\end{array}$ & $\begin{array}{l}1,16 \pm 0,43 \\
1,86 \pm 0,98\end{array}$ & 4,675 \\
\hline Domínio Desconexão/Rejeição & $\begin{array}{l}\text { Não clínico } \\
\text { Clínico }\end{array}$ & $\begin{array}{l}1,62 \pm 0,63 \\
2,61 \pm 0,80\end{array}$ & 7,116 \\
\hline Dependência/Incompetência & $\begin{array}{l}\text { Não clínico } \\
\text { Clínico }\end{array}$ & $\begin{array}{l}1,39 \pm 0,60 \\
2,20 \pm 0,95\end{array}$ & 5,177 \\
\hline Vulnerabilidade a dores e doenças & $\begin{array}{l}\text { Não clínico } \\
\text { Clínico }\end{array}$ & $\begin{array}{l}1,48 \pm 0,62 \\
2,85 \pm 1,16\end{array}$ & 7,447 \\
\hline Emaranhamento & $\begin{array}{l}\text { Não clínico } \\
\text { Clínico }\end{array}$ & $\begin{array}{l}1,39 \pm 0,82 \\
2,82 \pm 1,35\end{array}$ & 6,501 \\
\hline Fracasso & $\begin{array}{l}\text { Não clínico } \\
\text { Clínico }\end{array}$ & $\begin{array}{l}1,36 \pm 0,79 \\
1,85 \pm 0,92\end{array}$ & 2,922 \\
\hline $\begin{array}{l}\text { Domínio Autonomia e Desempenho } \\
\text { Prejudicados }\end{array}$ & $\begin{array}{l}\text { Não clínico } \\
\text { Clínico }\end{array}$ & $\begin{array}{l}1,41 \pm 0,58 \\
2,43 \pm 0,74\end{array}$ & 7,819 \\
\hline Subjugação & $\begin{array}{l}\text { Não clínico } \\
\text { Clínico }\end{array}$ & $\begin{array}{l}1,52 \pm 0,77 \\
2,52 \pm 1,20\end{array}$ & 5,039 \\
\hline Autossacrifício & $\begin{array}{l}\text { Não clínico } \\
\text { Clínico }\end{array}$ & $\begin{array}{l}2,64 \pm 1,15 \\
3,78 \pm 1,43\end{array}$ & 4,534 \\
\hline Domínio Orientação para o Outro & $\begin{array}{l}\text { Não clínico } \\
\text { Clínico }\end{array}$ & $\begin{array}{l}2,08 \pm 0,79 \\
3,15 \pm 1,14\end{array}$ & 5,557 \\
\hline Inibição emocional & $\begin{array}{l}\text { Não clínico } \\
\text { Clínico }\end{array}$ & $\begin{array}{l}1,89 \pm 1,04 \\
2,87 \pm 1,53\end{array}$ & 3,800 \\
\hline Padrões inflexíveis & $\begin{array}{l}\text { Não clínico } \\
\text { Clínico }\end{array}$ & $\begin{array}{l}2,72 \pm 1,04 \\
3,49 \pm 1,12\end{array}$ & 3,642 \\
\hline Domínio Supervigilância e Inibição & $\begin{array}{l}\text { Não clínico } \\
\text { Clínico }\end{array}$ & $\begin{array}{l}2,31 \pm 0,86 \\
3,18 \pm 1,03\end{array}$ & 4,733 \\
\hline Merecimento & $\begin{array}{l}\text { Não clínico } \\
\text { Clínico }\end{array}$ & $\begin{array}{l}2,05 \pm 1,01 \\
2,85 \pm 1,02\end{array}$ & 4,091 \\
\hline $\begin{array}{l}\text { Autocontrole e autodisciplina } \\
\text { insuficientes }\end{array}$ & $\begin{array}{l}\text { Não clínico } \\
\text { Clínico }\end{array}$ & $\begin{array}{l}1,89 \pm 1,07 \\
3,20 \pm 1,20\end{array}$ & 5,929 \\
\hline Domínio Limites Prejudicados & $\begin{array}{l}\text { Não clínico } \\
\text { Clínico }\end{array}$ & $\begin{array}{l}1,97 \pm 0,96 \\
3,03 \pm 0,93\end{array}$ & 5,757 \\
\hline
\end{tabular}




\section{Análise discriminante}

Os resultados do Coeficiente da função discriminante linear de Fisher, nos grupos clínico e não clínico, de acordo com os diferentes domínios do instrumento, podem ser observados na tabela 3.

De acordo com a tabela 4, pode-se perceber que 81,3\% dos grupos estão corretamente classificados. Também é possível perceber que, na população normal, 88,2\% foram corretamente classificados segundo o modelo, enquanto entre os alcoolistas apenas 75\%.

Em uma análise discriminante, deve-se avaliar a significância estatística das diferenças entre os grupos. Objetivando isso, existem quatro testes estatísticos (Traço de Hotelling's, Lambda de Wilks', Raiz Máxima de Roy's e Traço de Pillai's) que se destinam a recusar a hipótese nula (de que os grupos seriam iguais). O Traço de Pillai-Bartlett, também chamado de Critério de Pillai's, é o teste mais conservador, dentre os quatro mais utilizados em análises discriminantes, e avalia o poder discriminatório da função, se foi significativo ou não. Neste estudo, verifica-se que houve significância estatística quanto ao poder discriminatório da análise discriminante, uma vez que o valor de $p$ encontrado para os quatro testes foi de 0,000 . Os resultados podem ser observados pormenorizadamente na tabela 5.

Tabela 3. Classificação do coeficiente da função

\begin{tabular}{lcc}
\hline \multirow{2}{*}{ Domínios } & \multicolumn{2}{c}{ Grupo } \\
\cline { 2 - 3 } & Clínico & Não clínico \\
\hline Desconexão/Rejeição & 1.586 & 0,854 \\
Autonomia e desempenho prejudicados & 2.348 & 0,834 \\
Orientação para 0 outro & 0,120 & 0,189 \\
Supervigilância e inibição & 1.287 & 1.347 \\
Limites prejudicados & 1.387 & 0,892 \\
Constante & -9.961 & -4.610 \\
\hline Funcão discriminante linear de Fisher & &
\end{tabular}

Tabela 4. Classificação dos resultados

\begin{tabular}{llccc}
\hline \multirow{2}{*}{ Grupo } & \multicolumn{4}{c}{ Previsão de membro do grupo } \\
\cline { 2 - 5 } & & Nãoclínico & Clínico & Total \\
\hline Original & Não clínico & 45 & 6 & 51 \\
& Clínico & 14 & 42 & 56 \\
$\%$ & Não clínico & 88,2 & 11,8 & 100,0 \\
& Clínico & 25,0 & 75,0 & 100,0 \\
\hline
\end{tabular}

$81,3 \%$ do grupo original corretamente classificados.

Tabela 5. Testes multivariados

\begin{tabular}{lccc}
\hline Teste & Valor & $\mathbf{F}$ & Erro df \\
\hline Pillai's Trace & 0,405 & $13,722^{\mathrm{a}}$ & 101,000 \\
Wilks' Lambda & 0,595 & $13,722^{\mathrm{a}}$ & 101,000 \\
Hotelling's Trace & 0,679 & $13,722^{\mathrm{a}}$ & 101,000 \\
Roy's Largest Root & 0,679 & $13,722^{\mathrm{a}}$ & 101,000 \\
\hline aExact statistic $p=0,005, p=0,000$ & & &
\end{tabular}

\section{DISCUSSÃO}

Os resultados do YSQ-S2 demonstram que ocorreram diferenças estatisticamente significativas entre os grupos, tanto em domínios quanto em esquemas. Isso denota que o instrumento se mostra sensível para identificar diferenças entre grupos clínicos e não clínicos, corroborando o estudo holandês que também compara população clínica versus população não clínica, demonstrando estabilidade e poder discriminativo do instrumento. A análise discriminante apresentada em um estudo realizado na Holanda sugere alta sensibilidade do YSQ-S2 e suas subescalas na predição à presença ou ausência de psicopatologia. Nesse sentido, a amostra clínica holandesa obteve escores significativamente mais altos do que a não clínica para todas as subescalas ${ }^{14}$.

Ball et al..$^{20}$ avaliaram sintomas psiquiátricos, problemas psicossociais, resposta ao tratamento e transtornos de personalidade em 52 pacientes abusadores de substância internados para tratamento. Em metade da amostra identificou-se o uso de álcool e, desses, 85\% preencheram critérios para dependência, e a outra metade tinha as drogas ilícitas (23\% cocaína, 14\% heroína e 14\% maconha) como principal substância de escolha. Os autores acima citados haviam previsto que o domínio desconexão/rejeição seria proeminente em uma amostra cuja vida parecia caracterizada por temas de abandono/instabilidade, desconfiança/abuso, privação emocional, isolamento social e defectividade/vergonha. Desses cinco esquemas, apenas o isolamento social se destacou mais; dois dos mais elevados esquemas (autossacrifício e autocontrole/autodisciplina insuficientes) não eram previsíveis e apareceram com pontuações significativas.

Futuros estudos, na percepção de Harris e Curtin ${ }^{21}$, deveriam empregar amostras clínicas ou em situação de risco para avaliar mudanças na sintomatologia e nos EID. Na amostra que compõe esse estudo, os esquemas autossacrifício e autocontrole/autodisciplina insuficientes e isolamento social também obtiveram diferenças, como no estudo citado anteriormente, o que pode ser associado à dificuldade de controlar o uso da substância, assim como ao frequente abuso como estratégia de socialização. Pinto-Gouveia et al.22 mostraram que os pacientes com fobia social pontuaram mais alto do que a população não clínica em todos os esquemas. Os autores assinalam que os EID estão relacionados com uma percepção pelo self como um fracasso, socialmente inábil, indesejável e isolado em pacientes com outros transtornos de ansiedade.

A avaliação da estrutura fatorial do YSQ finlandês foi realizada por Saariaho et al..$^{23} \mathrm{em}$ amostras de pacientes com dor crônica e grupos controles com análise fatorial confirmatória. Nos resultados, os alfas individuais para as subescalas que avaliam os EID variaram entre a 0,94 e a 0,79 no grupo dos pacientes com dor e entre a 0,94 e a 0,81 no grupo controle. Em todos os casos, esses níveis foram bem acima do a 0,70. 
Os resultados demonstraram que a estrutura fatorial proposta por Young et al. ${ }^{4}$ também pode ser confirmada com a versão finlandesa da escala e em pacientes com sintomas somáticos. Os achados acima explicitados mostraram um nível satisfatório de consistência interna da escala, confirmando os resultados encontrados em outros países ${ }^{5,24-27}$. Exceto para o esquema dependência/incompetência $(0,566)$, todos os demais apresentaram níveis satisfatórios de consistência interna, que vão de 0,719 a 0,905, corroborando os achados deste estudo.

De acordo com Riso et al.28, em uma amostra de 100 pacientes deprimidos, dos 16 EID, 15 tiveram maiores correlações com os mesmos no seguimento, do que com qualquer outro esquema. Os autores também sugerem que é importante analisar a estabilidade dos EID em outras populações clínicas e em pessoas com menos condições crônicas. Soygüt et al. ${ }^{6}$ compararam, usando o teste $t$, a pontuação de populações clínicas e populações normais no YSQ e observaram diferenças estatisticamente significativas em alguns esquemas e domínios. Os autores concluem que os achados evidenciam que a estrutura fatorial do instrumento é geralmente consistente de acordo com estudos anteriores e que têm níveis aceitáveis de confiabilidade e validade. Além disso, quando os grupos foram comparados, observou-se que a amostra clínica obteve escores mais altos em todas as subescalas do que a amostra normal, o que vai ao encontro dos achados nessa amostra.

As análises de regressão hierárquica revelaram neste estudo que as percepções da infância, abuso emocional e negligência emocional continuaram a exercer influência sobre os sintomas posteriores e foram associadas mais tarde com sintomas de ansiedade e depressão, mediadas por esquemas de vulnerabilidade, vergonha e autossacrifício ${ }^{29}$.

O YSQ tem sido demonstrado como sensível para acessar crenças fundamentais, ou EID, das populações clínicas ${ }^{30}$. Em uma pesquisa com 137 pacientes clínicos, foram encontradas correlações significativas entre as escalas do YSQ. Lee et al..$^{27}$ aplicaram o teste t e encontraram escores significativamente mais altos para quase todos os fatores replicáveis de personalidade quando comparados a pacientes com transtornos em eixo l.

Nesse estudo, cabe ressaltar que se identificou como limitação o fato de não ter sido aplicada nenhuma escala para avaliar possíveis transtornos de humor, apenas distinguindose a população geral por ser ou não abusadora/dependente de álcool.

\section{CONCLUSÃO}

A partir das evidências da literatura que corroboram os resultados obtidos nessa amostra, pode-se observar que o YSQ-S2 é um instrumento capaz de detectar diferenças entre populações clínicas e não clínicas, sendo fundamental em uma avaliação mais profunda do funcionamento cognitivo do paciente. Para afirmações mais abrangentes, sugere-se a partir dos resultados do YSQ-S2 futuros estudos para confirmar os achados nessa população clínica, com amostras maiores, e/ou com outras populações clínicas.

\section{AGRADECIMENTOS}

À bolsista de iniciação científica Paola Lucena dos Santos, pela dedicação ao trabalho.

Apoio: Conselho Nacional de Desenvolvimento Científco e Tecnológico (CNPq).

\section{REFERÊNCIAS}

1. Young JE. Cognitive therapy for personality disorder. A schema-focused approach. Professional Resource Exchange, Inc, Sarasota, FL; 1990.

2. Young JE. Cognitive therapy for personality disorder. A schema-focused approach. $2^{\text {nd }}$ ed. Professional Resource Exchange, Inc, Sarasota, FL; 1994.

3. Young JE, Klosko JS, Weishaar ME. Terapia do esquema: guia de técnicas cognitivo-comportamentais inovadoras. New York: The Guilford Press; 2008.

4. Young JE, Klosko JS, Weishaar ME. Schema Therapy: a practitioner's guide. New York: The Guilford Press; 2003.

5. Schmidt NB, Joiner Jr TE, Young JE, Telch MJ. The Schema Questionnaire: investigation of psychometric properties and the hierarchical structure of a measure of maladaptive schemas. Cogn Ther Res. 1995;19(3):295-321.

6. Soygüt $G$, Karaosmanoğlu A, Çakir Z. Assessment of early maladaptive schemas: a psychometric study of the Turkish young schema questionnaire-short form-3. Turk Psikiyatri Derg. 2009;20(1):75-84.

7. Cazassa MJ, Oliveira MS. Terapia focada em esquemas: conceituação e pesquisas. Rev Psiq Clín. 2008;35(5):187-95.

8. Cazassa MJ, Oliveira MS. Validação brasileira do Questionário dos Esquemas. Estud Psicol. (PUCCAMP. Impresso, no prelo).

9. Masson 0, Plattes H, Tyson M. Early maladaptative schemas and adult attachment in a UK clinical population. Psychol Psychother. 2005;78(Pt4):549-64.

10. Jovev M, Jackson HJ. Early maladaptive schemas in personality disordered individuals. J Pers Disord. 2004;18(5):467-78.

11. Unoka Z, Tölgyes T, I Czobor P. Early maladaptive schemas and body mass index in subgroups of eating disorders: a differential association. Compr Psychiatry. 2007;48(2):199-204.

12. Stopa L, Waters A. The effect of mood on responses to the Young Schema Questionnaire: short form. Psychol Psychother. 2005;78(1):45-57.

13. Specht MW, Chapman A, Cellucci T. Schemas and borderline personality disorder symptoms in incarcerated women. J Behav Ther Exp Psychiatry. 2009;40(2):256-64.

14. Anderson K, Rieger $E$, Caterson I. A comparison of maladaptive schemata in treatment-seeking obese adults and normal-weight control subjects. J Psychosom Res. 2006;60(3);245-52.

15. Calvete E, Estévez A, Arroyabe EL, Ruiz P. The Schema Questionnaire-Short Form: structure and relationship with automatic thoughts and symptoms of affective disorders. EJPA. 2005;21(2):90-9.

16. Rijkeboer MM, Bergh H, Bout J. Stability and discriminative power of the Young SchemaQuestionnaire in a Dutch clinical versus non-clinical population. J Behav Ther Exp Psychiatry. 2005;36(2):129-44.

17. Bailleux S, Romo L, Kindynis S, Radtchenko A, Debray Q. Study of the bonds between early maladaptive schemas and strategies of coping (among alcohol-dependent patients and depressed patients). JTCC. 2008;18(1):19-25.

18. Brotchie J, Meyer C, Copello A, Kidney R, Waller G. Cognitive representations in alcohol and opiate abuse: The role of core beliefs. Br J Clin Psychol. 2004;43(Pt 3);337-42. 
19. Bisquerra R, Sarriera JC, Martínez F. Introdução à estatística: enfoque informático com o pacote estatístico SPSS. Porto Alegre: ArtMed; 2004.

20. Ball SA, Cobb-Richardson P, Connolly AJ, Bujosa CT, O'Neall TW. Substance abuse and personality disorders in homeless drop-in center clients: symptom severity and psychotherapy retention in a randomized clinical trial. Compr Psychiatry, 2005;46(5):371-9.

21. Harris AE, Curtin L. Parental perceptions, early maladaptive schemas, and depressive symptoms in young adults. Cognin Ther Res. 2002;26(3):405-16.

22. Pinto-Gouveia J, Castilho P, Galhardo A, Cunha M. Early maladaptive schemas and social phobia. Cogn Ther Res. 2006;30(5):571-84

23. Saariaho T, Saariaho A, Karila I, Joukama M. The psychometric properties of the Finnish Young Schema Questionnaire in chronic pain patients and a non-clinical sample. J Behav Ther Exp Psychiatry. 2009;40(1):158-68.

24. Baranoff J, Oei TPS, Cho SH, Kwon S. Factor structure and internal consistency of the Young Schema Questionnaire (Short Form) in Korean and Australian samples. J Affect Disord. 2006;93(1-3):133-40
25. Hoffart A, Sexton H, Hedley LM, Wang CE, Holthe H, Haugum JA, et al. The structure of maladaptive schemas: a confirmatory factor analysis and a psychometric evaluation of factor-derived scales. Cogn Ther Res. 2005;29(6):627-44.

26. Waller $G$, Meyer C, Ohanian V. Psychometric properties of the long and short versions of the Young Schema Questionnaire: core beliefs among bulimic and comparison women. Cogn Ther Res. 2001;25(2):137-47.

27. Lee $C$, Taylor $G$, Dunn J. Factor structure of the schema questionnaire in a large clinical sample. Cogn Ther Res. 1999;23(4):441-51.

28. Riso LP, Froman SE, Raouf M, Gable P, Maddux RE, Turini-Santorelli N, et al. The long-term stability of early maladaptive schemas. Cogn Ther Res. 2006;30(4):515-29.

29. Wright MO, Crawford E, Del Castillo D. Childhood emotional maltreatment and later psychological distress among college students: the mediating role of maladaptive schemas. Child Abuse Negl. 2009;33(1):59-68.

30. Dutra L, Callahan K, Forman E, Mendelsohn M, Herman J. Core schemas and suicidality in a chronically traumatized population. J Nerv Ment Dis. 2008;196(1):71-4. 